\title{
PERCEPÇÕES ACERCA DO PROGRAMA PET-SAÚDE/GRADUASUS NA FORMAÇÃO DO PROFISSIONAL FISIOTERAPEUTA: UM ESTUDO TRANSVERSAL QUANTITATIVO
}

\author{
PERCEPTIONS ON THE WORK PROGRAM PET-SAÚDE/GRADUASUS \\ ON PHYSICAL THERAPY SKILLS DEVELOPMENT: \\ A QUANTITATIVE, CROSS-SECTIONAL STUDY
}

\begin{abstract}
RESUMO
A Fisioterapia sempre teve sua atuação focada no âmbito reabilitador e curativo, mas programas como o PET-Saúde/ GraduaSUS têm permitido a inclusão os estudantes na Atenção Básica $(\mathrm{AB})$, para que um novo olhar de atuação já comece a ser construído desde a graduação. O presente estudo teve como objetivo avaliar a percepção e o perfil de acadêmicos de Fisioterapia acerca das vivências experienciadas no PET-Saúde/GraduaSUS. Utilizou-se metodologia quantitativa transversal com análise de dados. Obteve-se uma mostra de 28 petianos de 5 Universidades Federais, distribuídos entre as regiões Sul/Sudeste (14), Norte/Nordeste (8) e Centro-Oeste (6) e participantes do programa; apresentaram uma idade média de 22,64 anos $( \pm 2,26)$, sendo a grande maioria mulheres $(75 \%)$. De acordo com o desenvolvimento do projeto, os petianos afirmaram que as competências da Fisioterapia estão sendo desenvolvidas na $\mathrm{AB}$, assim como os objetivos propostos pelo programa, mas houve diferenças entre as regiões. Os pontos negativos apontados foram a estrutura da Unidade Básica de Saúde e sua adequação à Fisioterapia; por fim, afirmaram que o programa contribui para sua formação, proporcionando melhor conhecimento e olhar diferenciado em relação à atuação da Fisioterapia na AB. Portanto, apesar das barreiras ainda encontradas, a Fisioterapia vem-se fazendo cada vez mais presente na AB, e programas como o PET-Saúde/GraduaSUS têm ajudado a inserir esse profissional nesse nível de atenção desde a graduação, além de promover uma formação diferenciada.

Palavras-chave: Fisioterapia; Sistema Único de Saúde; Atenção Primária à Saúde.
\end{abstract}

Contato

Silvia Lanziotti Azevedo da Silva

E-mail: silviafisiojf@yahoo.com.br

${ }^{1}$ Programa de Residência Multiprofissional em Saúde da Família, Universidade Federal de Alfenas,

Minas Gerais, Brasil.

${ }^{2}$ Instituto de Ciências da Motricidade, Universidade Federal de Alfenas,

Minas Gerais, Brasil.

\section{ABSTRACT}

Physiotherapy has always been focused on rehabilitation and healing, but programs such as PET-Saúde / GraduaSUS have allowed students to be included in Primary Care (AB), so that a new approach can be built from graduation. This study aims to assess the perception and profile of Physiotherapy students about the experiences experienced at PET-Saúde / GraduaSUS. Cross-sectional quantitative methodology was used with data analysis. A sample of 28 students from 5 Federal Universities, distributed among the South / Southeast (14), North / Northeast (8), and Midwest (6) regions and program participants were obtained; they presented a mean age of 22.64 years $( \pm 2.26)$, the majority being women (75\%). According to the development of the project, the petits affirmed that the competences of Physiotherapy are being developed in $\mathrm{AB}$, as well as the objectives proposed by the program, but there were differences between the regions. The negative points pointed out were the structure of the Basic Health Unit and its adequacy to Physiotherapy; Finally, they affirmed that the program contributes in their formation, providing better knowledge and a differentiated look in relation to the performance of Physiotherapy in AB. Therefore, in spite of the barriers still found, Physiotherapy has become increasingly present in AB, and programs such as PET-Saúde / GraduaSUS have helped the insertion of such professional in this level of attention since graduation, in addition to promoting a differentiated training.

Keywords: Physical therapy specialty; Unified Health System; Primary health care. 


\section{INTRODUÇÃO}

$\mathrm{Na}$ atualidade, temos o Sistema Único de Saúde (SUS) como um dos maiores sistemas de saúde pública do mundo ${ }^{1}$. Com o avanço do sistema nos últimos anos, tornou-se necessária a formação de profissionais da área da saúde capacitados para atuação no SUS e dentro da Rede de Atenção à Saúde (RAS), principalmente na Atenção Básica $(\mathrm{AB})$, potencializando sua capacidade coordenadora de cuidado e sua resolutividade ${ }^{2}$. Apesar dessa nova lógica, os sistemas de ensino superior, nas áreas de saúde, ainda são muito voltados para o modelo biomédico ${ }^{3}$. Eles configuram-se como inadequados para o enfrentamento dos desafios da atenção à saúde, já que a ênfase curricular é dada ao tratamento de manifestações de doenças, com atuação do profissional voltada para a atenção individual, e não coletiva, e priorização da formação especializada ${ }^{4}$.

Dentro desse contexto, a Fisioterapia, em toda sua história, sempre foi vista como uma profissão de atuação reabilitadora e curativa ${ }^{4}$. A criação do Núcleo Ampliado de Saúde da Família e Atenção Básica (Nasf), em $2008^{5}$, foi o grande passo para a diversificação das atividades do fisioterapeuta além do caráter reabilitador forte na atenção especializada, uma vez que inseriu a profissão na $\mathrm{AB}$. Entretanto, a inclusão do fisioterapeuta na $\mathrm{AB}$ não foi acompanhada por mais discussões sobre sua atuação nesse nível de atenção na graduação em Fisioterapia na maioria das universidades ou faculdades, o que pode afastar o profissional do novo trabalho proposto $^{6}$.

Em busca de incentivar a formação para o SUS e para a AB, uma alternativa para os estudantes de Fisioterapia foi a inserção destes em projetos submetidos a editais de Programas de Educação para o Trabalho em Saúde (PET-Saúde) ${ }^{7}$, que incluem estes estudantes na $\mathrm{AB}$, buscando um novo olhar de atuação, desde a graduação. Em 2015, foi lançado o edital do PET-Saúde/GraduaSUS ${ }^{8}$, com uma proposta de trabalho interdisciplinar, desenvolvimento de ações interprofissionais aplicadas à comunidade, promoção da interação entre ensino, pesquisa e extensão, de modo a contribuir para o desenvolvimento sociocultural e científico dos acadêmicos ${ }^{9}$. O projeto incentiva também a integração ensino-serviço-comunidade, a institucionalização das atividades pedagógicas dos profissionais do serviço e a contribuição para a mudança curricular alinhada às Diretrizes Curriculares $\mathrm{Na}$ cionais(DCN) para os cursos de graduação na área da saúde ${ }^{4}$.

Diante da abrangência do projeto e dos seus objetivos de mudança na formação dos profissionais de saúde, o presente estudo visa avaliar a percepção de estudantes de fisioterapia petianos, seu perfil e como suas vivências no programa podem contribuir para sua formação, tornando-os profissionais que visem além dos horizontes tradicionais preconizados na formação, abrindo novas possibilidades e estabelecendo perspectivas maiores de atuação. 


\section{METODOLOGIA}

A presente investigação trata-se de um estudo quantitativo transversal. A população do estudo foi constituída por discentes de Fisioterapia de universidades federais brasileiras, que foram contempladas pelo programa PET-Saúde/GraduaSUS. Foram incluídos alunos bolsistas e voluntários, de ambos os sexos, de todas as idades e em qualquer período do curso. Para garantir uma maior padronização da amostra e por serem em número pequeno, foram excluídas as universidades particulares e estaduais, mesmo que possuíssem grupos da Fisioterapia aprovados.

Primeiramente, em cada universidade, foi feito contato com docente tutor/coordenador responsável pelo grupo da Fisioterapia, que autorizou a participação dos alunos. Essa autorização foi enviada por e-mail, com assinatura e carimbo, questionário e modelo de autorização, os quais também se disponibilizariam a passar o e-mail dos alunos participantes do programa.

Do total de 13 universidades federais, obteve-se contato e se dispuseram a participar do estudo mediante autorização prévia 5 instituições, distribuídas entre as regiões Sul/Sudeste (2), Norte/ Nordeste (2) e Centro-Oeste (1).

Após autorização do tutor, o projeto foi enviado ao Comitê de Ética em Pesquisa da Unifal-MG e aprovado pelo parecer número 2.195.544. Em seguida, foi realizado contato por e-mail com os alunos participantes do PET- Saúde/GraduaSUS, em que estes foram informados sobre o estudo e convidados a participar da pesquisa.
Os que concordaram em participar, receberam por e-mail um link que os direcionava a uma aba do Google Docs, contendo o Termo de Consentimento Livre e Esclarecido (TCLE), acompanhado do Questionário Geral estruturado, autoaplicável e com respostas automaticamente gravadas; Para esse contato, foi criada uma conta de e-mail exclusiva para o projeto, controlada pela pesquisadora responsável.

O questionário geral avaliava questões de caracterização da amostra, a atuação do aluno dentro das possibilidades oferecidas pela sua instituição e sobre a atuação especifica no PET-Saúde e suas atividades. Para caracterização da amostra, foram consideradas: idade, universidade e região do país onde a instituição é localizada. Em relação à atuação do aluno no curso, era questionado o período em que aluno se encontra, a(s) área(s) de interesse de atuação dentro da Fisioterapia, se é bolsista ou voluntário no PET-Saúde, se participa de outros projetos dentro da universidade e se também é ligado à Saúde Coletiva.

Especificamente relacionado com o PET-Saúde, foram avaliadas a percepção dos estudantes sobre as competências desenvolvidas, o cumprimento dos objetivos propostos pelo edital, condições de realização do trabalho proposto (carga horária, condições das Unidades Básicas de Saúde - UBS, adequação ao trabalho da Fisioterapia), barreiras encontradas dentro da Integração-Ensino-Serviço, conhecimento dos alunos sobre a atuação do fisioterapeuta na $\mathrm{AB}$ e suas expectativas futuras. 
Foi concedido aos participantes o prazo de um mês para responder ao questionário, e foi considerado um limite de cinco tentativas de contato por e-mail com os alunos. Nos casos em que não se obteve resposta, o discente foi excluído da pesquisa.

\section{Análise Estatística}

Para caracterização da amostra, foram realizadas análises de frequência absoluta e relativa para as variáveis categóricas, e de tendência central e dispersão para as variáveis contínuas.

As universidades foram agrupadas de acordo com as regiões onde eram localizadas, e os percentuais de resposta para cada questão foi comparado entre as regiões pelos Testes Qui-Quadrado de Pearson e Exato de Fisher, quando apropriado. Para todas as análises, foi considerado nível de significância $\alpha=0,05$, e foram realizadas no pacote estatístico $R$ 3.4.1.

\section{RESULTADOS}

O presente estudo contou com uma amostra de 28 petianos, divididos em três blocos regionais de acordo com a localização de sua universidade: metade é da região Sul/Sudeste (50\%); e os demais, das regiões Norte/Nordeste (29\%) e Centro-Oeste $(21 \%)$. Os participantes apresentaram uma idade média de 22,64 anos $( \pm 2,26)$, sendo maioria mulheres $(75 \%)$. Demais informações para caracterização da amostra estão presentes na tabela 1 .
Em relação à especialidade da Fisioterapia, baseado naquelas regulamentadas pelo Conselho Federal de Fisioterapia e Terapia Ocupacional (Coffito) ${ }^{10}$, metade dos petianos demonstraram interesse pela especialidade Saúde da Mulher, e os demais dividiram-se entre as outras áreas, dando destaque aos $14 \%$ que se consideravam generalistas.

Comparando os petianos das regiões definidas no estudo em relação às competências da Fisioterapia desenvolvidas dentro do trabalho do PET-Saúde/ GraduaSUS, os petianos, em todas elas, afirmaram que a maioria das competências estavam sendo total ou parcialmente desenvolvidas. Diferenças foram observadas na consideração do trabalho das práticas profissionais do estudante de Fisioterapia na $A B$, que foi maior nas regiões Norte/ Nordeste e Centro-Oeste $(\mathrm{p} \leq 0,001)$; no desenvolvimento do trabalho interdisciplinar, mais identificado pelos alunos da região Centro-Oeste $(p=0,001)$; e nas ações de promoção de saúde/prevenção de agravos com contribuição da Fisioterapia, que foram mais identificados como competência pelos alunos da região Norte/ Nordeste $(\mathrm{p}=0,007)$ (tabela 2$)$.

Embora as diferenças não tenham sido significativas entre as regiões estudadas ( $p>0,05)$, dentro das propostas que estavam no edital do programa, os estudantes identificaram as seguintes ações como desenvolvidas no PET-Saúde/ GraduaSUS: projetos/propostas que visem mudanças curriculares alinhadas às DCN (71\%); ampliação do SUS enquanto espaço de aprendizagem para estudantes e professores (92\%); divisão da responsabi- 
Tabela 1. Descrição dos participantes da pesquisa

\begin{tabular}{|c|c|c|}
\hline Variável & Frequência Absoluta & Frequência Relativa (\%) \\
\hline \multicolumn{3}{|l|}{ Sexo } \\
\hline Masculino & 7 & 25 \\
\hline Feminino & 21 & 75 \\
\hline \multicolumn{3}{|l|}{ Região } \\
\hline Sul/Sudeste & 14 & 50 \\
\hline Norte/Nordeste & 8 & 29 \\
\hline Centro-Oeste & 6 & 21 \\
\hline \multicolumn{3}{|l|}{ Período no curso } \\
\hline $1^{\circ}$ ao $4^{\circ}$ semestre & - & - \\
\hline $5^{\circ}$ ao $8^{\circ}$ semestre & 18 & 64 \\
\hline $9^{\circ}$ ao $10^{\circ}$ semestre & 10 & 36 \\
\hline \multicolumn{3}{|c|}{ Vínculo com o PET-Saúde/GraduaSUS } \\
\hline Bolsista & 16 & 57 \\
\hline Voluntário & 12 & 43 \\
\hline \multicolumn{3}{|c|}{ Vínculo com outros projetos } \\
\hline Sim & 21 & 75 \\
\hline Não & 7 & 25 \\
\hline \multicolumn{3}{|c|}{ Projetos relacionados com Saúde Coletiva } \\
\hline Sim & 8 & 29 \\
\hline Não & 20 & 71 \\
\hline
\end{tabular}

lidade pela saúde do território de atuação entre o Programa Saúde da Família (PSF) e a Instituição de Ensino (53,5\%); desenvolvimento do papel dos trabalhadores do SUS como principais responsáveis pela integração ensino-serviço-comunidade em conjunto com a Instituição de Ensino (71\%); e educação permanente em saúde de gestores, trabalhadores, professores, alunos e usuários (75\%), considerando para os percentuais a amostra.

Um ponto que apresentou diferenças entre as regiões foram as condições para a realização dos trabalhos propostos dentro do programa. Os petianos das regiões Norte/Nordeste e Centro-Oeste consideraram as UBS inadequadas para a realização do trabalho dos fisioterapeutas, em percentual superior ao considerado pelos estudantes da região Sul/Sudeste $(\mathrm{p}=0,011)$ (tabela 3$)$.
Sobre as barreiras que a Fisioterapia enfrenta para a integração ensinoserviço, também não houve diferenças significativas entra as regiões. Porém, quando considerada a "resistência dos usuários”, somente a região Norte/Nordeste (100\%) não identificou esse ponto como barreira (tabela 4).

Por fim, em relação ao conhecimento dos petianos sobre a atuação da Fisioterapia na $\mathrm{AB}$ e o interesse deles por essa área, os estudantes das regiões Sul/ Sudeste e Norte/Nordeste relataram mais conhecimento e interesse ( $\mathrm{p} \leq 0,001)$, embora na região Centro-Oeste todos relataram que o fisioterapeuta está presente nesse primeiro nível de atenção, na rede municipal $(\mathrm{p}=0,003)$ (tabela 5). 
Tabela 2. Competências da Fisioterapia desenvolvidas dentro do trabalho do PET-Saúde/ GraduaSUS

\begin{tabular}{|c|c|c|c|c|c|}
\hline \multirow{2}{*}{ Variáveis } & Total & Sul/Sudeste & Norte/Nordeste & Centro-Oeste & \multirow[b]{2}{*}{$p$} \\
\hline & n (\%) & n (\%) & n (\%) & n (\%) & \\
\hline \multicolumn{6}{|c|}{ Prática profissional do estudante de Fisioterapia na AP } \\
\hline Sim & $27(97 \%)$ & $13(93 \%)$ & $8(100 \%)$ & $6(100 \%)$ & \\
\hline Não & - & - & - & - & $\leq 0,001$ \\
\hline Parcialmente & $1(3 \%)$ & $1(7 \%)$ & - & - & \\
\hline \multicolumn{6}{|c|}{ Efetivação da integração Ensino-Serviço } \\
\hline Sim & $22(79 \%)$ & $12(86 \%)$ & $4(50 \%)$ & $6(100 \%)$ & \\
\hline Não & - & - & - & - & 0,261 \\
\hline Parcialmente & $6(21 \%)$ & $2(14 \%)$ & $4(50 \%)$ & - & \\
\hline \multicolumn{6}{|c|}{ Desenvolvimento de trabalho interdisciplinar } \\
\hline Sim & $25(89 \%)$ & $13(93 \%)$ & $6(75 \%)$ & $6(100 \%)$ & \\
\hline Não & - & - & - & - & 0,001 \\
\hline Parcialmente & $3(11 \%)$ & $1(7 \%)$ & $2(25 \%)$ & - & \\
\hline \multicolumn{6}{|c|}{ Ações de promoção de saúde ou promoção de agravos com participação da Fisioterapia } \\
\hline Sim & $23(82 \%)$ & $11(79 \%)$ & $8(100 \%)$ & $4(67 \%)$ & \\
\hline Não & - & - & - & - & 0,007 \\
\hline Parcialmente & $5(18 \%)$ & $3(21 \%)$ & - & $2(33 \%)$ & \\
\hline \multicolumn{6}{|c|}{ Contribuição para reestruturação dos currículos do curso de Fisioterapia } \\
\hline Sim & $22(79 \%)$ & $11(79 \%)$ & $5(62,5 \%)$ & $6(100 \%)$ & \\
\hline Não & $4(14 \%)$ & $3(21 \%)$ & $1(12,5 \%)$ & - & 0,232 \\
\hline Parcialmente & $2(7 \%)$ & - & $2(25 \%)$ & - & \\
\hline \multicolumn{6}{|c|}{ Produção acadêmica voltada para necessidade do serviço de Saúde para Fisioterapia } \\
\hline Sim & $17(61 \%)$ & $7(50 \%)$ & $6(75 \%)$ & $4(67 \%)$ & \\
\hline Não & $1(3 \%)$ & $1(7 \%)$ & - & - & 0,844 \\
\hline Parcialmente & $10(36 \%)$ & $6(43 \%)$ & $2(25 \%)$ & $2(33 \%)$ & \\
\hline \multicolumn{6}{|c|}{ Capacitação profissional para o serviço } \\
\hline Sim & $19(68 \%)$ & $12(86 \%)$ & $5(62,5 \%)$ & $2(33 \%)$ & \\
\hline Não & $2(7 \%)$ & - & $1(12,5 \%)$ & $1(17 \%)$ & 0,119 \\
\hline Parcialmente & $7(25 \%)$ & $2(14 \%)$ & $2(25 \%)$ & $3(50 \%)$ & \\
\hline \multicolumn{6}{|c|}{ Temas trabalhados relevantes para o trabalho do Fisioterapeuta } \\
\hline Sim & $22(79 \%)$ & $9(64 \%)$ & $8(100 \%)$ & $5(83 \%)$ & \\
\hline Não & - & - & - & - & 0,257 \\
\hline Parcialmente & $6(21 \%)$ & $5(36 \%)$ & - & $1(17 \%)$ & \\
\hline
\end{tabular}


Tabela 3. Condições para realização dos trabalhos propostos dentro do PET-Saúde/GraduaSUS

\begin{tabular}{|c|c|c|c|c|c|}
\hline \multirow{2}{*}{ Variáveis } & Total & Sul/Sudeste & Norte/Nordeste & Centro-Oeste & \multirow{2}{*}{$p$} \\
\hline & n (\%) & n (\%) & n (\%) & n (\%) & \\
\hline \multicolumn{6}{|c|}{ Carga horária de 8 horas/semanais } \\
\hline Adequada & $19(68 \%)$ & $10(72 \%)$ & $5(62,5 \%)$ & $4(67 \%)$ & \multirow{3}{*}{0,731} \\
\hline Excede & $3(11 \%)$ & $1(7 \%)$ & $2(25 \%)$ & - & \\
\hline Não consome & $6(21 \%)$ & $3(21 \%)$ & $1(12,5 \%)$ & $2(33 \%)$ & \\
\hline \multicolumn{6}{|c|}{ Condições da Unidade Básica de Saúde } \\
\hline Excelente/Muito boa & $9(32 \%)$ & $7(50 \%)$ & $1(12,5 \%)$ & $1(17 \%)$ & \multirow{3}{*}{0,152} \\
\hline Boa & $12(43 \%)$ & $6(14 \%)$ & $4(50 \%)$ & $2(33 \%)$ & \\
\hline Regular/Muito ruim & $7(25 \%)$ & $1(7 \%)$ & $3(37,5 \%)$ & $3(50 \%)$ & \\
\hline \multicolumn{6}{|c|}{ Adequação da Unidade Básica de Saúde para a Fisioterapia } \\
\hline Excelente/Muito boa & - & - & - & - & \multirow{3}{*}{0,011} \\
\hline Boa & $7(25 \%)$ & $5(36 \%)$ & $1(12,5 \%)$ & $1(17 \%)$ & \\
\hline Regular/Muito ruim & $21(75 \%)$ & $9(64 \%)$ & $7(87,5 \%)$ & $5(83 \%)$ & \\
\hline
\end{tabular}

Tabela 4. Barreiras para a Fisioterapia na integração ensino-serviço

\begin{tabular}{|c|c|c|c|c|c|}
\hline \multirow{2}{*}{ Varáveis } & Total & Sul/Sudeste & Norte/Nordeste & Centro-Oeste & \multirow{2}{*}{$p$} \\
\hline & n (\%) & n (\%) & n (\%) & n (\%) & \\
\hline \multicolumn{6}{|c|}{ Resistência dos usuários } \\
\hline Sim & $7(25 \%)$ & $5(36 \%)$ & - & $2(33 \%)$ & \\
\hline Não & $19(68 \%)$ & $8(57 \%)$ & $8(100 \%)$ & $3(50 \%)$ & 0,144 \\
\hline Não sei & $2(7 \%)$ & $1(7 \%)$ & - & $1(17 \%)$ & \\
\hline \multicolumn{6}{|c|}{ Resistência dos profissionais não participantes do PET } \\
\hline Sim & $13(46 \%)$ & $7(50 \%)$ & $3(37,5 \%)$ & $3(50 \%)$ & \\
\hline Não & $13(46 \%)$ & $6(43 \%)$ & $4(50 \%)$ & $3(50 \%)$ & 1,000 \\
\hline Não sei & $2(7 \%)$ & $1(7 \%)$ & $1(12,5 \%)$ & - & \\
\hline \multicolumn{6}{|c|}{ Relacionamento interpessoal } \\
\hline Sim & $11(39 \%)$ & $6(43 \%)$ & $2(25 \%)$ & $3(50 \%)$ & \\
\hline Não & $17(61 \%)$ & $8(57 \%)$ & $6(75 \%)$ & $3(50 \%)$ & 0,413 \\
\hline Não sei & - & - & - & - & \\
\hline \multicolumn{6}{|l|}{ Comunicação } \\
\hline Sim & $12(43 \%)$ & $8(57 \%)$ & $2(25 \%)$ & $2(33 \%)$ & \\
\hline Não & $16(57 \%)$ & $6(43 \%)$ & $6(75 \%)$ & $4(67 \%)$ & 0,378 \\
\hline Não sei & - & - & - & - & \\
\hline \multicolumn{6}{|c|}{ Perfil inadequado do estudante } \\
\hline Sim & $8(28 \%)$ & $5(36 \%)$ & $2(25 \%)$ & $1(17 \%)$ & \\
\hline Não & $17(61 \%)$ & $7(50 \%)$ & $6(75 \%)$ & $4(66 \%)$ & 0,707 \\
\hline Não sei & $13(10 \%)$ & $2(14 \%)$ & - & $1(17 \%)$ & \\
\hline \multicolumn{6}{|c|}{ Rigidez nas propostas de trabalho } \\
\hline Sim & $5(18 \%)$ & $3(22 \%)$ & $1(12,5 \%)$ & $1(17 \%)$ & \\
\hline Não & $21(75 \%)$ & $10(71 \%)$ & $7(87,5 \%)$ & $4(66 \%)$ & 0,860 \\
\hline Não sei & $2(7 \%)$ & $1(7 \%)$ & - & $1(17 \%)$ & \\
\hline \multicolumn{6}{|c|}{ Falta de conhecimento dos preceptores acerca da atuação na Fisioterapia na AB } \\
\hline Sim & $11(39 \%)$ & $6(43 \%)$ & $5(62,5 \%)$ & - & \\
\hline Não & $16(57 \%)$ & 7 (50\%) & $3(37,5 \%)$ & $6(100 \%)$ & 0,077 \\
\hline Não sei & $1(4 \%)$ & $1(7 \%)$ & - & - & \\
\hline
\end{tabular}


Tabela 5. Conhecimento dos "petianos” sobre a atuação da Fisioterapia na Atenção Básica

\begin{tabular}{|c|c|c|c|c|c|}
\hline \multirow{2}{*}{ Variáveis } & Total & Sul/Sudeste & Norte/Nordeste & Centro-Oeste & \multirow{2}{*}{$p$} \\
\hline & n (\%) & n (\%) & n (\%) & n (\%) & \\
\hline \multicolumn{6}{|c|}{ A Fisioterapia é inserida na $\mathrm{AB}$} \\
\hline Sim & $27(96 \%)$ & $14(100 \%)$ & $8(100 \%)$ & $5(83 \%)$ & \\
\hline Não & $1(4 \%)$ & - & - & $1(17 \%)$ & $\leq 0,001$ \\
\hline Não sei & - & - & - & - & \\
\hline \multicolumn{6}{|c|}{ Oportunidade de acompanhar a atuação deste profissional nesse nível de atenção } \\
\hline Sim & $20(71 \%)$ & $10(72 \%)$ & $4(50 \%)$ & $6(100 \%)$ & \\
\hline Não & $7(25 \%)$ & $3(21 \%)$ & $4(50 \%)$ & - & 0,197 \\
\hline Não sei & $1(4 \%)$ & $1(7 \%)$ & - & - & \\
\hline \multicolumn{6}{|c|}{ Existência de Fisioterapeuta na $\mathrm{AB}$ do município } \\
\hline Sim & $24(86 \%)$ & $11(79 \%)$ & $7(87,5 \%)$ & $6(100 \%)$ & \\
\hline Não & $4(14 \%)$ & $3(21 \%)$ & $1(12,5 \%)$ & - & 0,003 \\
\hline Não sei & - & - & - & - & \\
\hline \multicolumn{6}{|c|}{ Trabalho desenvolvido no PET-Saúde acrescenta para atuação como Fisioterapeuta na AB } \\
\hline Sim & $26(92 \%)$ & $13(93 \%)$ & $8(100 \%)$ & $5(83 \%)$ & \\
\hline Não & $1(4 \%)$ & - & - & $1(17 \%)$ & 0,463 \\
\hline Não sei & $1(4 \%)$ & $1(7 \%)$ & - & - & \\
\hline \multicolumn{6}{|c|}{ Pretende ou desenvolve TCC na área de AB/Saúde Coletiva/Saúde Pública } \\
\hline Sim & $9(32 \%)$ & $6(43 \%)$ & $2(25 \%)$ & $1(17 \%)$ & \\
\hline Não & $15(54 \%)$ & $7(50 \%)$ & $3(37,5 \%)$ & $5(83 \%)$ & 0,241 \\
\hline Não sei & $4(14 \%)$ & $1(7 \%)$ & $3(37,5 \%)$ & - & \\
\hline \multicolumn{6}{|c|}{ É importante a atuação do Fisioterapeuta na $\mathrm{AB}$} \\
\hline Sim & $28(100 \%)$ & $14(100 \%)$ & $8(100 \%)$ & $6(100 \%)$ & \\
\hline Não & - & - & - & - & NC \\
\hline Não sei & - & - & - & - & \\
\hline \multicolumn{6}{|c|}{ Pretende atuar como Fisioterapeuta na $\mathrm{AB}$} \\
\hline Sim & $13(46 \%)$ & $4(29 \%)$ & $7(87,5 \%)$ & $2(33 \%)$ & \\
\hline Não & $5(18 \%)$ & $3(21 \%)$ & - & $2(33 \%)$ & 0,087 \\
\hline Não sei & $10(36 \%)$ & $7(50 \%)$ & $1(12,5 \%)$ & $2(33 \%)$ & \\
\hline
\end{tabular}

NC: Não calculado

\section{DISCUSSÃO}

Embasados nos resultados do estudo, podemos identificar pontos destacados pelos estudantes em relação a sua própria formação, as competências desenvolvidas pelo PET-Saúde/GraduaSUS, bem como os desafios para atuação identificados e os conhecimentos acerca das possibilidades de atuação do fisioterapeuta na $A B$.
Em todas as regiões estudadas, as competências da Fisioterapia mais desenvolvidas foram a prática profissional do estudante, o desenvolvimento do trabalho interdisciplinar e a participação da Fisioterapia em ações que visam à promoção de saúde e prevenção de agravos. Esse resultado apresenta-se em consonância com o inciso I do art. $4^{\circ}$ das DCN do Curso de Graduação em Fisioterapia ${ }^{11}$, que cita: “os profissionais de saúde [...] devem estar 
aptos a desenvolver ações de prevenção, proteção, proteção e reabilitação da saúde, tanto em nível individual quanto coletivo"; e o inciso III do art. $5^{\circ}$, que afirma que a "formação do Fisioterapeuta deve capacitá-lo para saber atuar multiprofissionalmente, interdisciplinarmente e transdisciplinarmente, com extrema produtividade na promoção de saúde”. O PET-Saúde/GraduaSUS, dessa forma, pode ser considerado ferramenta de desenvolvimento de competências nos estudantes previstas pelas DCN, capaz de promover uma mudança no caráter de atuação do profissional. Embora o desenvolvimento de competências tenha sido identificado em todas as regiões, os tipos de competências foram diferentes entre elas. Uma explicação seriam possíveis caraterísticas próprias de cada grupo PET-Saúde. A documentação que regulamenta o Programa é vaga e superficial, o que facilita a operacionalização dentro de diversos cenários e realidades das instituições, o que permite que cada grupo construa seu percurso ${ }^{12}$, e apareçam as diferenças regionais, como essa encontrada no presente estudo.

A ação de maior destaque dentro das propostas do programa neste estudo foi a ampliação do SUS enquanto espaço de aprendizagem para estudantes e professores. O PET-Saúde é considerado uma forma de suprir a deficiência de aprendizado nas escolas de saúde no quesito formação para a $\mathrm{AB}$ e Estratégia Saúde da Família $(E S F)^{13}$. Ele é capaz de transformar esse cenário em local de produção e troca de conhecimentos, tanto para os alunos e professores quanto para os profissionais da $A^{13}$. Isso se encaixa no princípio de Educação Permanente, na qual os profissionais devem ser capazes de aprender continuamente, tanto na formação quanto na prática ${ }^{13}$.

Em contrapartida, a ação identificada como menos desenvolvida foi a divisão de responsabilidade pela saúde do território entre a ESF e demais profissionais da $A B$ e a Instituição de Ensino Superior (IES). Tal fato pode ser explicado pelas atuações finitas dos programas PETSaúde, prejudicando a criação de vínculo dos petianos alunos e tutores com os profissionais e usuários, prejudicando princípios da $A B$, como a longitudinalidade ${ }^{14}$. Mesmo diante desse achado, acredita-se que o PET-Saúde pode ser capaz de instigar o aluno a ter um olhar mais crítico sobre a saúde do território, criando assim um futuro profissional com o desejo de capacidade para atuar valorizando o vínculo entre profissional e comunidade, além da preocupação com questões sociais, políticas e humanitárias que permeiam o processo saúde-doença ${ }^{4}$.

Sobre as condições de trabalho para o desenvolvimento das atividades, poucos identificaram as UBS como adequadas para atuação do Fisioterapeuta, principalmente nas regiões Norte/ Nordeste e Centro-Oeste. Tal percepção pode ser devido à associação da atuação profissional fisioterapeuta às clínicas especializadas; e, diante disso, os estudantes não conseguem ver suas possibilidades de atuação dentro da estrutura da $\mathrm{AB}^{15}$. O uso de equipamentos é visto, historica- 
mente, como uma identidade profissional do fisioterapeuta, e existe uma valorização da tecnologia, das especialidades e das práticas direcionadas à assistência curativa $^{15}$. Entretanto, diante das tendências mais atuais de formação e ação em saúde, sabe-se que, quando inserido na $A B$, o fisioterapeuta pode ser de grande valia para ações de promoção da saúde, prevenção de doenças e educação em saúde, ações estas que não se limitam ao uso de equipamentos ou a um ambiente físico, mas na valorização do usuário e do contexto em que este encontra-se inserido ${ }^{16}$. Ao comparar as regiões, a percepção da adequação das UBS foi mais positiva na região Sul/Sudeste. De acordo com um estudo desenvolvido na Universidade Federal de Alagoas ${ }^{17}$, um dos grandes problemas da região Nordeste é a estrutura física da UBS, e tais limitações estruturais afetam a formação dos futuros profissionais que lá estão inseridos. Esse pode ser um dos fatores que levou à diferença identificada no estudo.

As barreiras para integração ensino-serviço da Fisioterapia descritas pelo presente estudo foram semelhantes em todas as regiões do País. A resistência dos profissionais pode estar relacionada com o fato das UBS onde são realizados os trabalhos já apresentarem suas próprias características, seu funcionamento, o que demanda uma adaptação por parte de alunos, professores, trabalhadores participantes e não participantes do $\mathrm{PE}$ T-Saúde, e principalmente dos usuários, cada um com características e pensamentos próprios ${ }^{18,19}$. Outra barreira que merece destaque é a realidade na qual não é comum o trabalho em equipe na rotina das Unidades; e para que este seja implementado, é necessário organização, amadurecimento do grupo ao partilhar conhecimentos, trabalho articulado e esclarecimento sobre as funções de cada profissional dentro da produção do cui$\operatorname{dado}^{16}$.

Em relação ao interesse dos alunos em aprofundar seus conhecimentos e possibilidade de continuidade da atuação na área, a maioria afirmou não saber se desenvolverá seu Trabalho de Conclusão de Curso ou trabalharão dentro da $\mathrm{AB}$, relatando ter interesse em outras especialidades. Esse resultado pode ser devido ao fato de a inserção da profissão ainda ser recente na $A B$, e muitas diretrizes curriculares ainda serem voltadas para uma formação especialista ${ }^{20}$. Contudo, o estudo demonstra que os petianos têm conhecimento da inserção do Fisioterapeuta e de sua atuação na $\mathrm{AB}$, e $14 \%$ se consideram generalistas, o que pode representar mudança e expansão nos cenários de atuação desse profissional. O principal fator responsável por essa visão dos petianos é o processo de mudança das DCN, que tem ocasionado a inclusão e/ou ampliação de disciplinas de ciências sociais e humanas e saúde coletiva nas grades curriculares, habilitando o futuro profissional a identificar aspectos humanos e sociais envolvidos no processo saúde-doença ${ }^{13,21}$.

A principal limitação do estudo foi a pequena adesão das universidades participantes, uma vez que foi alcançado contato com apenas 5 das 13 IES federais com grupos de fisioterapia do PET-Saúde/ GraduaSUS, o que limita uma maior visão da eficácia do programa. Ainda assim, foi possível identificar diferenças regionais, ponto abordado em poucos estudos que demonstrem as diferenças dos grupos PET de acordo com a região, enfatizando as especificidades de cada um, e permitindo uma visão em nível nacional. 


\section{CONCLUSÃO}

Apesar da identificação de barreiras relacionadas com a estrutura das unidades, bem como do pequeno relato de motivação para o trabalho no primeiro nível da Rede de Atenção como profissional, estudantes inseridos no Programa PET-Saúde/ GraduaSUS são capazes de reconhecer a importância da atuação do fisioterapeuta na $\mathrm{AB}$ e de valorizar ações de promoção de saúde e prevenção de agravos como ferramentas de atuação. As diferenças regionais relacionadas com o desenvolvimento de competência demonstram o caráter regionalizado do projeto, o que vai ao encontro da diversidade brasileira. Os resultados permitem afirmar que o PET-Saúde é uma ferramenta capaz de orientar uma nova formação de Fisioterapeutas e deve ser incentivado nas IES.

\section{REFERÊNCIAS}

1. Brasil. Lei no 8.080 , de 19 de setembro de 1990. Dispõe sobre as condições para a promoção, proteção e recuperação da saúde, a organização e o funcionamento dos serviços correspondentes e dá outras providências. Diário Oficial da União; 19 set.

2. Rodrigues AAAO. Processo de Interação Ensino, Serviço e Comunidade: a experiência de um PET-Saúde. Rev Bras Educ Med 2012; 36(1):184-192.

3. Machado MFAS, Monteiro EMLM, Quiroz, DT, Vieira, NFC, Barroso, MGT. Integralidade, formação de saúde, educação em saúde e as propostas do SUS- uma revisão conceitual. Ciênc Saúde Coletiva 2007;12(2):335-342.
4. Caldas JB, Lopes ACS, Mendonça RD, Figueiredo A, Lonts JGA, Ferreira EF, et al. A percepção de alunos quanto ao Programa de Educação pelo Trabalhado para a Saúde - PET-Saúde. Rev Bras Educ Med 2012; 36(1):33- 41.

5. Brasil. Ministério da Saúde, Gabinete do Ministro. Portaria ${ }^{\circ} 154$ de 24 de janeiro de 2008. Cria os Núcleos de Apoio à Saúde da Família. [acessado 2018 nov 23]. Disponível em: http://bvsms. saude.gov.br/bvs/saudelegis/gm/2008/ prt0154_24_01_2008.html.

6. Formiga NFB, Ribeiro KSQS. Inserção do Fisioterapeuta na Atenção Básica: uma Analogia entre Experiências Acadêmicas e a Proposta dos Núcleos de Apoio à Saúde da Família (NASF). Rev Bras Cienc Saúde 2012; 16(2):113-122.

7. Brasil. Ministério da Saúde. Gabinete do Ministro. Portaria Interministerial $\mathrm{n}^{\circ}$ 1.802 , de 26 de agosto de 2008. Institui o Programa de Educação pelo Trabalho para a Saúde - PET-Saúde. [acessado 201823 nov]. Disponível em: http:// bvsms.saude.gov.br/bvs/saudelegis/ gm/2008/pri1802_26_08_2008.html.

8. Brasil. Secretária de Gestão do Trabalho e da Educação na Saúde. Edital no 13, de 2015. Seleção para o Programa de Educação pelo Trabalho para Saúde PETSaúde/GraduaSUS- 2017/ 2017. Diário Oficial da União 2015; 29 set.

9. Tanaka EE. Projeto PET-Saúde: Ferramenta de Aprendizado na Formação Profissional em Saúde. Rev Bras Educ Med 2012; 36(1):136-140.

10. Especialidades Fisioterapia reconhecidas pelo Conselho Federal de Fisioterapia Terapia Ocupacional [internet]. [acessado 201826 nov]. Disponível em: https://www.coffito.gov.br/nsite/?page_ id $=2350$. 
11. Brasil. Conselho Nacional de Educação. Câmara de Educação Superior. Resolução CNE/CES 4, de 19 de fevereiro de 2002. Institui Diretrizes Curriculares Nacionais do Curso de Graduação em Fisioterapia. [acessado 201817 nov] Disponível em: http:// portal.mec.gov.br/cne/arquivos/pdf/ CES042002.pdf.

12. Fonseca GS, Junqueira SR. Programa de Educação pelo Trabalho para a Saúde da Universidade de São Paulo (Campus Capital): o olhar dos tutores. Ciênc Saúde Coletiva 2014; 19(4):11511162.

13. Barbosa EL, Carvalho TPV, Júnior MBG, Araújo KCG. Fisioterapia Além das especialidades: conhecimento do alunato sobre a inserção do fisioterapeuta na saúde coletiva. Cad Edu, Saude e Fis 2015; 2(3):40-51.

14. Freitas PH. Repercussões do PETSaúde na formação de estudantes da área de saúde. Esc Anna Nery 2013; 17(3):496-504.

15. Lima AJ, Aley NRL, Brito GEG, Goyatá SLT, Silva SLA. Resolutividade da Fisioterapia na Atenção Básica à Saúde $(\mathrm{AB})$ : a percepção de fisioterapeutas. Cad Educ Saude Fis 2017; 4(8):14-22.
16. Silva DJ, Ros MA. Inserção de profissionais de fisioterapia na equipe de saúde da família e Sistema Único de Saúde: desafios na formação. Ciênc Saúde Coletiva 2007; 12(6):1673-1681.

17. Cavalcante MVS, Lima TCS. A precarização do trabalho na atenção básica em saúde: relato de experiência. Argumentum 2013; 5(1):235-256.

18. Sobrinho TAO, Medeiros CPP, Maia MR, Reis TC, Miranda LP, Costa PF Integração acadêmica e multiprofissional no PET-Saúde: Experiências e Desafios. Revista ABENO 2011; 11(1):39-42.

19. Castardeli E, Castardeli C, Pinto TR. O programa de Educação pelo Trabalho para Saúde como porta de entrada para o protagonismo da docência na saúde. Rev. Bras. Pesq. Saúde 2015; 17(2):98102 .

20. Pizzinato A. A integração EnsinoServiço como estratégia na formação profissional para o SUS. Rev Bras Educ Med 2012; 36(1-2):170-177.

21. Souza JO, Oliveira BC A influência do PET-Saúde na formação do estudante de Fisioterapia: relato de experiência. Ciênc Saúde Coletiva 2015; 8(2):94-77. 